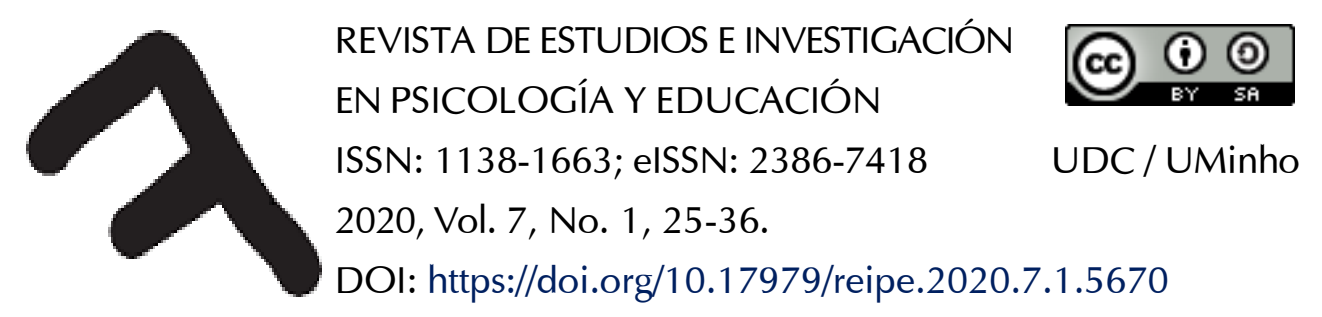

\title{
Determinantes individuais e organizacionais na percepção de indisciplina em sala de aula: Um estudo transcultural
}

\section{Individual and organisational determinants of perceptions of classroom indiscipline: A cross-cultural study}

\author{
Odete Cadete (iD*, Célia Oliveira (iD**, João Lopes (iD* \\ *Universidade do Minho, **Universidade Lusófona do Porto
}

\begin{abstract}
Resumo
A indisciplina nas escolas e os comportamentos desafiadores dos alunos constituem uma das principais preocupações dos professores, administradores e pais. Esta investigação visa comparar as percepções de indisciplina em salas de aula do ensino primário e secundário em estabelecimentos de ensino públicos e privados de Angola e Portugal. Participaram no estudo 1600 professores: 800 professores angolanos (493 homens e 307 mulheres) e 800 professores portugueses (181 homens e 619 mulheres). Os professores angolanos reportaram como ocorrendo com maior frequência comportamentos como intervir fora de vez, agressão verbal e desrespeito ao professor, enquanto os professores portugueses indicaram o comportamento de desatenção como sendo o mais frequente. As percepções de indisciplina diferem também em função do tipo de escola, do género e experiência profissional dos professores. Considerando que Angola saiu recentemente de décadas de guerra e enfrenta problemas cujo impacto na disciplina nas salas de aula não é suficientemente conhecido, pretende-se que este estudo seja inovador em Angola e que possa assumir uma dimensão transcultural, nomeadamente através da comparação de algumas variáveis e tipos de indisciplina com países como Portugal.
\end{abstract}

Palavras-chave: determinantes de indisciplina; professores; estudo transcultural

\begin{abstract}
Student indiscipline and misbehaviour are one of the biggest concerns of teachers, school managers and parents. The aim of this study is to compare perceptions of classroom indiscipline in public and private primary and secondary schools in Angola and Portugal. The survey group for the study included 1600 teachers: 800 Angolan teachers (493 men, 307 women) and 800 Portuguese teachers (181 men, 619 women). Angolan teachers reported a higher frequency of certain types of behaviour, such as talking out of turn, verbal aggression and disrespect towards teachers, while Portuguese teachers reported inattention as the most common behaviour. Perceptions of indiscipline differed according to the type of school and the teacher's gender and professional experience. Angola is a country that has recently emerged from decades of war and, as such, faces problems whose impact on classroom discipline is not yet fully known. This study offers new insight into the situation in Angola by providing a cross-cultural comparison of different variables and types of discipline with countries such as Portugal.
\end{abstract}

Keywords: determinants of indiscipline; teachers; cross-cultural study

Odete Cadete (D000-0001-7763-961X, João Lopes (D000-0001-5005-120X: Universidade do Minho. Campus de Gualtar. Rua da Universidade 4710 - 057 Braga, Portugal.

Célia Oliveira (D000-0002-8252-7337: Universidade Lusófona do Porto. Rua Augusto Rosa, nº 24, 4000-098 Porto, Portugal.

Correspondência relativa a este artigo: Odete Cadete - detecadete@ hotmail.com 
A indisciplina nas escolas e os comportamentos desafiadores dos alunos constituem uma das principais preocupações dos professores e de toda a comunidade escolar (Landrum, Lingo, \& Scott, 2011; Simón \& Alonso-Tapia, 2016). A indisciplina pode ser definida como o conjunto de comportamentos verbais e não verbais diversos, que prejudicam o normal funcionamento da sala de aula (Aires, 2010; Doyle, 1986). Diversos autores indicam como comportamentos disruptivos perturbadores do regular funcionamento de uma aula: recusa na realização de actividades, verbalizações desadequadas ao contexto em forma e modo (insultos; desrespeito); comportamentos que mobilizam a atenção dos colegas; assédio aos colegas (Hagenauer, Hascher, \&Volet, 2012; Lopes, 2006). Nash, Schlösser e Scarr (2016) conceptualizam os comportamentos indisciplinados como qualquer comportamento fora da tarefa (off-task) em sala de aula, que distraia o professor e/ou outros alunos do objectivo, ou seja, do ensino e aprendizagem.

Os professores consideram estes comportamentos como intoleráveis e geradores de stress, exigindo um esforço acrescido no processo de ensino-aprendizagem e gestão de sala de aula. Na sua percepção, as situações mais graves contêm um elemento de agressividade, principalmente quando dirigida a si (Amado \& Freire, 2009; Buchanan, 2010; Johnson \& Fullwood, 2006; Reynolds, Stephenson, \& Beaman, 2011). Aproximadamente um terço dos professores referem que os comportamentos disruptivos interferem no ensino (Robers, Zhang, \& Truman, 2012), diminuindo a sua eficácia, com prejuízo para a aprendizagem dos alunos (Aloe, Amo, \& Shaahan, 2014; Ferreira, dos Santos, \& Rosso, 2016; Johnson \& Fullwood, 2006). Além disso, dificultam a adaptação do aluno ao contexto educativo, contribuindo para a exaustão emocional dos professores e restantes intervenientes do contexto educativo (Hakanen, Bakker, \& Schaufeli, 2006; Piwowar, Thiel, \& Ophardt, 2013; Skaalvik \& Skaalvik, 2008, 2010; Tsouloupas, Carson, Matthews, Grawitch, \& Barber, 2010).

Estudos anteriores indicam como determinantes da indisciplina em sala de aula factores escolares (inerentes ao tipo de ensino, funcionamento da escola e clima organizacional), do professor (características pessoais e profissionais como experiência e capacidade de gestão de sala de aula) e familiares (Lopes, 2006; Renca, 2008; Teixeira, 2007).

Skaalvik e Skaalvik (2011) encontraram uma associação significativa entre a indisciplina e satisfação dos professores com o trabalho, por um lado, e a motivação para abandonar a profissão, por outro. Esta possibilidade de mudança profissional surge associada à rotatividade inerente à mesma e à exaustão emocional (Goddard, O’Brien, \& Goddard, 2006; Singh \& Billingsley, 1998; Tsouloupas et al., 2010). O desgaste emocional foi identificado na generalidade dos professores, desde os que iniciam a carreira aos mais experientes (Carson, 2011; Dicke, Parker, Marsh, Mareike, \& Detlev, 2014).

\section{Determinantes da indisciplina}

Os determinantes de indisciplina acima mencionados referem-se a determinantes organizacionais (tipo de escola) e as determinantes individuais ( género do professor e experiência profissional).

O tipo de escola, pública ou privada, em que os professores leccionam aparenta ter uma influência importante sobre a percepção de indisciplina. Diversos estudos referem que as escolas públicas apresentam mais indisciplina do que as escolas privadas (LeBlanc, Swisher, Vitaro, \& Tremblay, 2007). Estas últimas tendem, concomitantemente, a reportar menos comportamentos problemáticos e melhores rendimentos dos alunos (LeBlanc et al., 2007). Rothstein, Carvov e Benveiste (1999) encontraram também diferenças entre escolas públicas e privadas, salientando que as escolas privadas têm um sentido maior de descrição no que concerne a decisões administrativas, apresentando menor probabilidade de conflitos, uma vez que há maior homogeneidade entre os pais, e não responsabilizando os professores e pessoal administrativo pelas políticas públicas de hierarquia e burocracia.

A literatura aborda ainda a percepção de indisciplina e a variação de acordo com o género do professor. Professores do género masculino tendem a avaliar os comportamentos dos alunos de forma menos positiva, comparativamente a professores do género feminino (Hopf \& Hatzichristou, 1999), as quais parecem 
estabelecer maior empatia com os alunos (Yilmaz \& Sahinkaya, 2010). No entanto, parece ser importante ter em consideração o nível de ensino na análise destas diferenças. Hopf e Hatzichristou (1999) verificaram que em níveis de ensino mais elementares, professores do género feminino avaliavam a indisciplina dos alunos como sendo menos problemática. Contudo, à medida que os níveis escolares iam progredindo, eram os professores do género masculino a avaliar os comportamentos de indisciplina como menos problemáticos.

Outras variáveis, como o tipo de comportamento de indisciplina, parecem desempenhar também um papel diferente na visão dos professores. Gotzens, Badia, Genovard e Dezcallar (2010) constataram que professores do género masculino entendem como mais problemáticos comportamentos de indisciplina de nível social, tais como o uso de linguagem inadequada, agressão física (e.g., bater ou empurrar os colegas), roubo, entre outros, ao passo que para professores do género feminino parecem ser os comportamentos de nível instrucional, como por exemplo desobediência, intervir fora da vez, chegar atrasado a aula e a desatenção, que são mais graves.

O tempo de experiência profissional parece também ser factor importante na percepção da indisciplina do aluno, conforme mostra a literatura ao comparar professores em início de carreira com professores com mais experiência profissional. Alguns autores (Martin \& Baldwin, 1994; Polat, Kaya, \&Akdağ, 2013) referem que professores em início de carreira são menos eficazes na gestão de sala de aula, possuem menor autonomia no controlo de comportamentos indisciplinados, com tendência para serem mais autoritários em relação a regras e consequências, apresentando mais dificuldades em sala de aula. Estes profissionais, além de estarem a vivenciar os desafios inerentes ao início de suas carreiras, encontram-se muitas vezes em fase inicial no que concerne à constituição de famílias, tendo por isso um acréscimo de responsabilidades. Os profissionais em início de carreira demostram assim menor eficácia, quando comparados com professores com mais experiência profissional, que aparentam possuir mais estratégias na gestão da sala de aula e maior eficácia no controlo de comportamentos indisciplinados dos alunos na sala de aula (Özben, 2010).

Adicionalmente, os professores menos experientes gastam mais tempo a lidar com a indisciplina (Dalgiç \& Bayhan 2014). Por sua vez, os professores mais experientes olham para os comportamentos indisciplinados como sendo nocivos para os alunos, sobrecarregando-os com menos castigos, tendo mais conversas de forma individual com os alunos, e conseguindo melhor adesão dos alunos na aprendizagem (Espinosa, Garzón, \& Noguera, 2016).

\section{Comparação de Angola e Portugal na percepção de indisciplina}

De acordo com a literatura especializada (e.g., Chiu \& Chow, 2011; Ding, Li, Li, \& Kulm, 2008; Kyriacou \& Martin, 2010), um factor fundamental que parece ter influência ao nível da indisciplina e suas definições é a cultura do país. Esta diz respeito a um conjunto de factores envolventes da organização escolar, que incluem a restante comunidade educativa, tais como valorização do ambiente escolar, valorização social dos professores, contribuição da comunidade para a escola, entre outros. Para além de factores meramente sociodemográficos, a importância que se atribui à comunidade educativa e os laços comunitários em torno da escola variam de sociedade para sociedade, de meio urbano para meio rural, e espelham a percepção da sociedade acerca do papel da escola, integrada numa comunidade específica.

Alguns autores (Biggs, 1998; Lewis, Romi, Qui, \& Katz, 2005) referem que a cultura tem impacto ao nível da disciplina de escolas e salas de aulas em diversos países, sugerindo por exemplo que aspectos culturais como a ênfase dada ao colectivismo ou individualismo podem explicar os diferentes níveis de indisciplina encontrados em diferentes países. Neste sentido, alguns autores (Chiu \& Klassen, 2009; Sandberg \& Hoffert, 2001) indicaram que em países mais ricos os pais têm frequentemente um nível de escolaridade superior e passam mais tempo com os filhos, ensinando-lhes deste modo melhores competências sociais e autodisciplina. Por outro lado, em países mais pobres, os pais, apresentando um nível de escolaridade inferior e não tendo tanto tempo para despender com os filhos, apoiam-se mais nos professores para ensinarem disciplina adequada na sala de aula. 


\section{Objectivos do estudo}

O presente estudo tem como objectivos:

1. Comparar as percepções de indisciplina em salas de aula do ensino primário ao ensino secundário em escolas públicas e privadas de Angola e Portugal;

2. Analisar o efeito do tipo de escola na percepção de indisciplina em sala de aula;

3. Analisar o efeito de variáveis relacionadas com o professor, como o género e a experiência profissional, na percepção de indisciplina em sala de aula.

\section{Método}

\section{Participantes}

Participaram neste estudo 800 professores angolanos, 493 (61.6\%) do género masculino e 307 (38.4\%) do género feminino, e 800 professores portugueses, 181 (22.6\%) do género masculino, sendo a maioria do género feminino $(n=619,77.4 \%)$. A tabela 1 apresenta as medidas de estatística descritiva relativas à formação inicial, bem como as características do exercício da profissão.

\section{Tabela 1}

Medidas de estatística descritiva relativas a características no exercício da profissão dos professores angolanos e portugueses

\begin{tabular}{lcc}
\hline & Professores angolanos & Professores portugueses \\
$n(\%)$ & $(\%)$ \\
\hline Grau de curso de formação inicial & & $57(7.2)$ \\
Bacharelato & $158(19.9)$ & $674(84.8)$ \\
Licenciatura & $496(62.5)$ & $64(8.1)$ \\
Mestrado & $35(4.4)$ & $0(0.0)$ \\
Pós-graduação & $5(0.6)$ & $0(0.0)$ \\
Outro & $100(12.6)$ & $101(12.6)$ \\
Tempo de serviço (anos) & & $317(39.6)$ \\
Até 9 anos & $497(62.2)$ & $382(47.8)$ \\
10-20 anos & $263(32.9)$ & $511(67.8)$ \\
Mais de 20 anos & $39(4.9)$ & $243(32.2)$ \\
Situação profissional & & \\
Quadro efectivo & $501(63.3)$ & $594(78.8)$ \\
Contrato & $290(36.7)$ & $160(21.2)$ \\
Tipo de escola onde lecciona & & \\
Pública & $403(56.2)$ & \\
Privada & $314(43.8)$ & \\
\hline
\end{tabular}

\section{Instrumentos}

Para avaliar as diferentes variáveis do presente estudo foi utilizado o questionário de Indisciplina percebida, que visa avaliar o grau de indisciplina percebida pelo professor na sua sala de aula. Este questionário foi adaptado para a população portuguesa por Lopes e Oliveira (2016), a partir do questionário desenvolvido por Sun e Shek (2012), os quais através de entrevistas acederam aos comportamentos considerados problemáticos pelos professores. A escala de resposta deste questionário é de cinco pontos (1 - Raramente ou Nunca; 2 Poucas Vezes; 3 - Algumas Vezes; 4 - Muitas Vezes; 5 - Frequentemente). O mesmo foi aplicado também para a população angolana. Este questionário é composto por 25 questões, divididos em sete partes, e foram respondidas por professores do ensino primário e secundário de Angola e Portugal. Trata-se de um questionário em que cada questão tem um valor em si mesma, sendo assim que os dados são apresentados.

Para este estudo foram usadas especificamente algumas variáveis do questionário, que correspondem aos dados demográficos, a primeira parte do questionário, com 10 questões, sendo analisadas neste estudo 
especificamente três: o género e experiência profissional, designadas determinantes do professor, e o tipo de escola, que foi designado como determinante organizacional.

A segunda parte foi constituída por três questões. Nesta segunda parte averigua-se a frequência e a gravidade de 15 comportamentos perturbadores por parte dos alunos (e.g. "Intervir fora da vez (ex. cantar, ter conversas inapropriadas, fazer comentários inapropriados, gritar)"), bem como a percentagem de tempo de aula gasto com os mesmos (e.g., " $40 \%$ a 50\%). A escala de resposta à questão da frequência percebida de comportamentos inadequados é de cinco pontos, em que 1 corresponde a "Raramente ou nunca" e 5 corresponde a "Frequentemente". No que concerne à questão sobre a gravidade dos comportamentos inadequados, a escala de resposta é de cinco pontos, em que 1 corresponde a "Nada problemático" e 5 corresponde a "Extremamente Problemático".

As outras variáveis deste questionário não são do âmbito deste estudo, pelo que não se detalham neste artigo.

\section{Procedimentos}

No que diz respeito à recolha de dados relativos aos professores angolanos, o contacto junto das escolas foi efectuado através de uma carta dirigida ao Sr. Ministro da Educação de Angola, para autorização formal para realização do estudo e acesso directo às direcções de escolas públicas e privadas, contacto com as direcções de escolas (Directores e Professores) e apresentação do estudo à direcção de cada instituição, onde a solicitação formal antecedeu um encontro presencial entre a investigadora e os responsáveis pela instituição. Foi feita uma primeira aplicação piloto do questionário sobre indisciplina em contexto de sala de aula junto da populaçãoalvo, para determinar eventuais necessidades de alteração do mesmo. Foram tidos os cuidados prévios com a compreensão da linguagem usada no questionário e com o entendimento dos objectivos e âmbito em cada questão.

Após a análise dos resultados do estudo piloto, foi dado início à aplicação formal dos questionários. A aplicação de questionários nas escolas decorreu no período de um ano. Os questionários foram respondidos por professores pertencentes a escolas públicas e privadas Angolanas. Foram recolhidos 800 questionários. A recolha de dados junto dos professores portugueses foi precedida também de autorizações prévias junto da Direcção-Geral da Educação e das Direcções de agrupamentos de escolas de todo o território português, sendo solicitado às direcções que divulgassem o link que dava acesso ao questionário aos professores dos seus estabelecimentos de ensino. Foi disponibilizado um endereço de e-mail caso os participantes pretendessem apresentar dúvidas.

\section{Procedimentos de análise de dados}

A análise de dados foi realizada com recurso ao programa da IBM: Statistical Package for the Social Science (SPSS), versão 23. Para a análise descritiva são apresentadas frequências absolutas (n) e medianas (Mdn) e Amplitude interquartil (AIQ) para variáveis ordinais. Foi utilizado o teste de Mann-Whitney para comparar dois grupos independentes, e foi utlizado o Coeficiente de Correlação de Spearman para as análises de correlação.

\section{Resultados}

\section{Percepção de indisciplina em professores angolanos e portugueses}

A Tabela 2 apresenta os resultados das análises (U de Mann-Whitney) de diferenças relativas aos tipos de comportamentos de indisciplina, de acordo com a perspectiva dos professores angolanos e portugueses.

Foram encontradas diferenças estatisticamente significativas em relação a todos os comportamentos de indisciplina, à excepção do comportamento de brincar/fazer palhaçadas. Os professores angolanos percebem uma maior frequência de todos os tipos de comportamentos, quando comparados com professores portugueses, sendo que apenas no que se refere ao comportamento de desatenção se verifica o oposto. 
Tabela 2

Diferenças relativas aos tipos de comportamentos de indisciplina, de acordo com a perspectiva dos professores angolanos e portugueses

\begin{tabular}{lccc}
\hline & $\begin{array}{c}\text { Professores angolanos } \\
M d n(A I Q)\end{array}$ & $\begin{array}{c}\text { Professores portugueses } \\
M d n(A I Q)\end{array}$ & $U$ \\
\hline Intervir fora de vez & $3.00(2.00)$ & $3.00(2.00)$ & $290970.00^{*}$ \\
Agressão verbal & $3.00(3.00)$ & $2.00(2.00)$ & $249073.50^{*}$ \\
Desrespeito ao professor & $2.00(3.00)$ & $2.00(2.00)$ & $249590.50^{*}$ \\
Desatenção & $3.00(1.00)$ & $4.00(2.00)$ & $184607.00^{*}$ \\
Agressão física & $2.00(1.00)$ & $1.00(1.00)$ & $149235.50^{*}$ \\
Copiar trabalhos de casa & $3.00(2.00)$ & $2.00(2.00)$ & $181717.50^{*}$ \\
Brincar/fazer palhaçadas & $3.00(2.00)$ & $3.00(1.00)$ & 312158.00 \\
Chegar atrasado à aula & $3.00(2.00)$ & $2.00(1.00)$ & $205567.00^{*}$ \\
\hline
\end{tabular}

Nota: Mdn = Mediana; $A I Q=$ Amplitude Interquartil

$* p \leq .001$

\section{Percepção de indisciplina em função de variáveis organizacionais (tipo de escola), em professores angolanos e portugueses}

A Tabela 3 apresenta os resultados das análises de diferenças relativas aos tipos de comportamentos de indisciplina, de acordo com a variável organizacional "tipo de escola", para professores angolanos e portugueses.

\section{Tabela 3}

Diferenças na percepção de indisciplina em função do tipo de escola, em professores angolanos e portugueses

\begin{tabular}{lcccccc}
\hline & \multicolumn{3}{c}{ Professores angolanos } & \multicolumn{3}{c}{ Professsores portugueses } \\
\cline { 2 - 7 } & \multicolumn{2}{c}{ Ordem Média } & \multicolumn{2}{c}{ Ordem Média } \\
\cline { 2 - 7 } & $\begin{array}{c}\text { Escola } \\
\text { pública }\end{array}$ & $\begin{array}{c}\text { Escola } \\
\text { privada }\end{array}$ & $U$ & $\begin{array}{c}\text { Escola } \\
\text { pública }\end{array}$ & $\begin{array}{c}\text { Escola } \\
\text { privada }\end{array}$ & $U$ \\
\cline { 2 - 7 } Intervir fora de vez & 300.88 & 432.69 & $39848.50^{* *}$ & 395.20 & 311.79 & $37007.00^{* *}$ \\
Agressão verbal & 312.93 & 411.40 & $44971.50^{* *}$ & 391.95 & 323.86 & $38937.00^{* *}$ \\
Desrespeito ao professor & 312.32 & 415.06 & $44727.50^{* *}$ & 393.28 & 318.91 & $38146.00^{* *}$ \\
Desatenção & 358.27 & 359.94 & 62975.00 & 394.54 & 314.24 & $37399.00^{* *}$ \\
Agressão física & 337.63 & 382.81 & $54853.50 *$ & 392.02 & 323.59 & $38895.00^{* *}$ \\
Copiar trabalhos de casa & 401.17 & 300.56 & $44934.00^{* *}$ & 381.30 & 363.40 & 45264.00 \\
Brincar/fazer palhaçadas & 310.59 & 419.83 & $43855.00^{* *}$ & 389.51 & 332.91 & $40386.00^{*}$ \\
Chegar atrasado à aula & 406.28 & 298.32 & $44216.00^{* *}$ & 391.84 & 324.27 & $39003.00^{* *}$ \\
\hline
\end{tabular}

$* p<.01, * * p<.001$

No que diz respeito a professores angolanos, foram encontradas diferenças estatisticamente significativas, de acordo com o tipo de escola (pública ou privada), em relação a todos os tipos de comportamentos, excepto em relação à desatenção. Professores de escolas públicas reportam frequência superior de comportamentos de copiar os trabalhos de casa e chegar atrasado à aula; todos os outros comportamentos são referidos como tendo maior frequência na perspectiva de professores de escolas privadas.

Relativamente aos professores portugueses, foram encontradas diferenças estatisticamente significativas em função do tipo de escola em relação a todos os tipos de comportamentos, à excepção de copiar os trabalhos de casa. Verifica-se que professores de escolas públicas reportam uma frequência superior dos comportamentos de indisciplina.

\section{Percepção de indisciplina e variáveis sociodemográficas do professor, em professores angolanos e portugueses}

As Tabelas 4 e 5 apresentam os resultados das análises relativas aos tipos de comportamentos de indisciplina, de acordo com variáveis sociodemográficas do professor, para professores angolanos e portugueses. 
Tabela 4

Diferenças na percepção de indisciplina em função do género, em professores angolanos

\begin{tabular}{lccc}
\hline & $\begin{array}{c}\text { Masculino } \\
\text { Ordem Média }\end{array}$ & $\begin{array}{c}\text { Feminino } \\
\text { Ordem Média }\end{array}$ & $U$ \\
\hline Intervir fora de vez & 387.14 & 420.72 & $69088.00^{*}$ \\
Agressão verbal & 376.71 & 430.82 & $64408.50^{* *}$ \\
Desrespeito ao professor & 378.77 & 431.45 & $65192.50^{* *}$ \\
Desatenção & 419.92 & 369.32 & $66103.50^{* *}$ \\
Agressão física & 387.27 & 418.01 & 69155.50 \\
Copiar trabalhos de casa & 438.72 & 334.09 & $55262.00^{* *}$ \\
Brincar/fazer palhaçadas & 377.67 & 435.79 & $64535.50^{* *}$ \\
Chegar atrasado à aula & 432.22 & 346.60 & $59049.50^{* *}$ \\
\hline
\end{tabular}

${ }^{*} p<.05, * * p \leq .001$

Foram encontradas diferenças estatisticamente significativas entre professores angolanos do sexo masculino e feminino, ao nível da percepção de todos os comportamentos de indisciplina em estudo, à excepção da agressão física. Verifica-se que professores do sexo masculino percebem uma maior frequência dos comportamentos de desatenção, copiar os trabalhos de casa e chegar atrasado à aula, sendo que os restantes comportamentos são entendidos como mais frequentes por professores do sexo feminino (Tabela 4).

Não foram encontradas diferenças estatisticamente significativas em função do género, no que se refere aos professores portugueses.

\section{Tabela 5}

Relação entre percepção de indisciplina e o tempo de experiência profissional dos professores, para professores angolanos e professores portugueses

\begin{tabular}{lcc}
\hline & Professores angolanos & Professores portugueses \\
& $r$ & $r$ \\
\hline Intervir fora de vez & $-.37^{* *}$ & $-.09 *$ \\
Agressão verbal & $-.42^{* *}$ & -.04 \\
Desrespeito ao professor & $-.37^{* *}$ & -.01 \\
Desatenção & .07 & -.05 \\
Agressão física & $-.22^{* *}$ & -.00 \\
Copiar trabalhos de casa & $.38^{* *}$ & .05 \\
Brincar/fazer palhaçadas & $-.37^{* *}$ & $-.10^{*}$ \\
Chegar atrasado à aula & $.35^{* *}$ & .00 \\
\hline
\end{tabular}

Nota: Coeficiente de correlação de Spearman

${ }^{*} p<.05, * * p \leq .001$

Relativamente ao tempo de experiência (Tabela 5), no caso dos professores angolanos, verificou-se que um tempo de experiência superior estava significativamente relacionado com uma percepção de menor frequência de comportamentos de intervir fora de vez, agressão verbal, desrespeito ao professor, agressão física e brincar/fazer palhaçadas, e com maior frequência de comportamentos de copiar os trabalhos de casa e chegar atrasado à aula. No que se refere aos professores portugueses, um tempo de experiência superior revelou estar significativamente correlacionado com percepção de menor frequência de apenas dois tipos de comportamentos: intervir fora de vez e brincar/fazer palhaçadas.

\section{Discussão}

Os resultados encontrados mostraram diferenças entre os dois países em termos do tipo de comportamentos de indisciplina, na medida em que professores angolanos percepcionam uma maior frequência de comportamentos, como intervir fora de vez, agressão verbal e desrespeito, à excepção do comportamento de desatenção, que é percepcionado como ocorrendo mais vezes pelos professores portugueses. Chiu e Chow 
(2011) sugerem que as características dos países podem afectar a disciplina em sala de aula, bem como mapear tipos de indisciplina, em função das características das escolas, tendo em consideração os respectivos regulamentos. De acordo com Lopes e Santos (2013), estas diferenças entre países, ao nível da disciplina em sala de aula, parecem ser mais qualitativas do que quantitativas.

Relativamente ao efeito de variáveis organizacionais, como o tipo de escola, na percepção de indisciplina em sala de aula, verificou-se que tanto em Portugal como em Angola há diferenças significativas entre ensino público e privado. A literatura sugere que nas escolas privadas os códigos de disciplina são dados a conhecer desde o início a alunos e pais, com regras claras quanto aos modelos a adoptar (De Regt \& Weenink, 2005), tendendo a referir menos comportamentos problemáticos (e.g., LeBlanc et al., 2007). Esta realidade é no entanto contrária à realidade angolana, em que se verificou uma maior frequência de alguns comportamentos problemáticos, como intervir fora de vez ou agressão verbal, em escolas privadas. Este facto pode estar relacionado com a falta de controlo por parte dos profesores em relação aos alunos, uma vez que os alunos dificilmente são sancionados pelos comportamentos indisciplinados e, por possuirem um nivel sócioeconómico superior ao dos alunos de escolas públicas, podem inclusive com muita facilidade optar por mudar para outras escolas do ensino privado sem dificuldades de vagas. Por outro lado, no ensino público angolano o número de alunos por turma é bastante elevando, dificultando assim a obtenção de vagas.

Santos e Pascoinho (2020) referem que a falta de padronização de medidas de irradicação da indisciplina na sala de aula por parte dos docentes promove o aumento de comportamentos indisciplinados, devido a ausência de uniformização no processo de reporte destes comportamentos. De acordo com Ferrinho (2012), a ausência de códigos de conduta mais explícitos, poderá afectar o desempenho organizacional, uma vez que os alunos não reconhecem um modelo uniforme na atenção dada pelos professores aos factores de indisciplina em sala de aula, o que revela ausência de estratégia organizacional. Na mesma linha, Tauber (2007) refere que regras pouco claras podem contribuir para o aumento de comportamentos indisciplinados dos alunos na sala de aula e levar a que alguns alunos, de forma intencional, testem os limites da organização, interferindo deste modo com as actividades de ensino e aprendizagem.

Relativamente ao efeito de variáveis relacionadas com os professores, como o género e experiência profissional, na percepção de indisciplina em sala de aula, constatou-se que os professores angolanos do género feminino percepcionam uma maior frequência de comportamentos como agressão verbal, desrespeito ao professor, agressão física, e brincar e fazer palhaçadas. Por outro lado, professores do género masculino percepcionam uma maior frequência de comportamentos como copiar os trabalhos de casa e chegar atrasado à aula. No caso dos professores portugueses, não foram encontradas diferenças significativas contrariamente aos professores angolanos em que os professores do género feminino percepcionam a generalidade dos comportamentos mais problemáticos. Estas diferenças parecem ser coerentes com resultados de estudos anteriores que sugerem que o género dos professores influencia os julgamentos que estes realizam acerca da gravidade dos problemas de comportamento dos alunos (e.g., Borg, 1998).

No que respeita ao tempo de experiência dos professores, constatou-se também que um tempo de experiência superior estava associado com percepção de maior frequência de alguns comportamentos, como copiar os trabalhos de casa, no caso dos professores angolanos, e com menor frequência de outros comportamentos, como intervir fora de vez, de acordo com professores dos dois países. É possível que professores mais experientes consigam, de forma mais rápida e eficaz, fazer cessar e controlar a longo prazo os comportamentos inapropriados dos alunos, tal como sugere a literatura (e.g., Özben, 2010; Tsouloupas, Carson, \& Mathews, 2014).

\section{Limitações do estudo e sugestões para futuras investigações}

Apesar de termos adoptado uma metodologia quantitativa, com uma amostra de dimensão significativa, os resultados deste estudo apenas consideraram a percepção dos professores, não tendo sido realizada uma observação "in loco" da realidade da sala de aula e as respostas foram obtidas por auto-relato, portanto não podem ser generalizados para além da própria amostra. 
De referir também o facto de este estudo utilizar dois métodos diferentes de recolha dos dados, sendo que para os professores portugueses a recolha foi realizada por meio digital online, e para os professores angolanos a recolha foi realizada em papel. O método de recolha online não permite deste modo um controlo do ambiente onde os participantes responderam ao questionário, porém constitui um método de recolha mais eficaz porque permite obter maior número de respostas de forma mais célere, contrariamente ao método de resposta em suporte de papel, que obrigou a deslocações presenciais e acaba por ser mais oneroso.

Será importante também estudar o efeito de outras variáveis como o número de alunos por turma e o efeito da variável eficácia colectiva na percepção de indisciplina em sala de aula.

\section{Referencias}

Aires, L. (2010). Disciplina na sala de aulas - Um guia de boas práticas para professores do $3^{\circ}$ ciclo do Ensino Básico e Ensino Secundário. Lisboa: Edições Sílabo.

Aloe, A., Amo, L., \& Shaahan, M. (2014). Classroom management self-efficacy and burnout: A multivariate meta-analysis. Educational Psychology Review, 26(1),101-126. https://doi.org/10.1007/s10648-013-9244-0

Amado, J. S., \& Freire, I. (2009). A(s) indisciplina(s) na escola: Compreender para prevenir. Coimbra: Almedina.

Biggs, J. (1998). Assessment and classroom learning: a role for summative assessment? Assessment in Education: Principles, Policy \& Practice, 5(1), 103-110. https://doi.org/10.1080/0969595980050106

Borg, S. (1998). Teachers' pedagogical systems and grammar teaching: A qualitative study. TESOL quarterly, 32(1), 9-38. https://doi.org/10.2307/3587900

Buchanan, D. A., \& Huczynski, A. A. (2010). Organizational behaviour. Pearson Education.

Carson, R. L., Plemmons, S., Templin, T. J., \& Weiss, H. M. (2011). "You are who you are:" A mixed-method study of affectivity and emotion regulation in curbing teacher burnout. In G. M. Reeve \& E. Frydenberg (Eds.), Personality, stress and coping. Implications for education (pp. 239-264). Charlotte, NC: Information Age Publishing.

Chiu, M. M., \& Chow B.Y. (2011). Classroom discipline across forty-one countries: School, economic, and cultural differences. Journal of Cross-Cultural Psychology, 42(3) 516-533. https://doi.org/10.1177/00220 22110381115

Chiu, M. M., \& Klassen, R. M. (2009). Calibration of reading self-concept and reading achievement among 15-year-olds: Cultural differences in 34 countries. Learning and Individual Differences, 19(3), 372-386. https://doi.org/10.1016/j.lindif.2008.10.004

Cohn, A. \& Maréchal, M. A. (2015). Laboratory measure of cheating predicts misbehavior at school. CESifo Working Paper Series No. 5613. Available at SSRN: https://ssrn.com/abstract=2706473

Dalgiç, G., \& Bayhan, G. (2014). A Meta-Analysis: Student misbehaviors that affect classroom management. Cypriot Journal of Educational Sciences, 9(2), 101-116.

De Regt, A., \& Weenink, D. (2005). When negotiation fails private education as a disciplinary strategy. Journal of Education Policy, 20(1), 59-80. https://doi.org/10.1080/0268093042000322838

Dicke, T. P., Parker, P., Marsh, H., Mareike, K. A. S., \& Detlev, L. (2014). Self-efficacy in classroom management, classroom disturbances, and emotional exhaustion: A moderated mediation analysis of teacher candidates. Journal of Educational Psychology, 106(2), 569-583. https://doi.org/10.1037/a0035504

Ding, M., Li, Y., Li, X., \& Kulm, G. (2008). Chinese teachers' perceptions of students' classroom misbehavior. Educational Psychology, 28(3), 305-324. https://doi.org/10.1080/01443410701537866

Doyle, W. (1986). Classroom organization and management. In M. C. Wittrock (Ed.), Handbook of research on teaching (pp. 392-431). New York: Macmillan.

Gil, F. J., Chillón, P., \& Delgado, M. Á. (2016). Gestión de aula ante conductas contrarias a la convivencia en Educación Secundaria Obligatoria (Classroom management inproblematic behaviors of coexistence in mandatory secondary education). Retos, 30, 48-53. https://recyt.fecyt.es/index.php/retos/article/view/42015 
Ferreira, A. C., dos Santos, E. R., \& Rosso, A. J. (2016). Representação social da indisciplina escolar. Psicologia: Teoria e Pesquisa, 32(1). https://doi.org/10.1590/0102-37722016012074199208

Ferreirinho, M. T. N. (2012). Indisciplina na sala de aula: Que realidade? (estudo exploratório). Dissertação de Mestrado, Universidade Lusíada de Lisboa. Lusíada - Repositorio das Universidades Lusíadas: http://hdl.handle.net/11067/532

Goddard, R., O’Brien, P., \& Goddard, M. (2006). Work environment predictors of beginning teacher burnout. British Educational Research Journal, 32, 857-874. https://doi.org/10.1080/01411920600989511

Gotzens Busquets, C., Badía Martín, M., Genovard, C., \& Dezcallar, T. (2010). A comparative study of the seriousness attributed to disruptive classroom behaviors. Electronic Journal of Research in Educational Psychology, 8(1), 33-58. http://ojs.ual.es/ojs/index.php/EJREP/article/view/1399

Hagenauer, G., Hascher, T., \& Volet, S. (2012). Teacher emotions in the classroom: Associations with students' engagement, classroom discipline and the interpersonal teacher-student relationship. European Journal Psychology of Education, 30(4), 385-403. https://doi.org/10.1007/s10212-015-0250

Hakanen, J. J., Bakker, A. B., \& Schaufeli, W. B. (2006). Burnout and work engagement among teachers. Journal of School Psychology, 43(6), 495-513. https://doi.org/10.1016/j.jsp.2005.11.001

Hopf, D., \& Hatzichristou, C. (1999). Teacher gender-related influences in Greek schools. British. Journal of Educational Psychology, 68(1), 1-18. https://doi.org/10.1348/000709999157527

Johnson, H. L., \& Fullwood, H. L. (2006). Disturbing behaviors in the secondary classroom: How do general educators perceive problem behaviors? Journal of Instructional Psychology, 33(1), 20-39.

Kyriacou, C., \& Martin, J. L. O. (2010). Beginning secondary school teachers' perceptions of pupil misbehavior in Spain. Teacher Development, 14(4), 415-426. https://doi.org/10.1080/13664530.2010.533481

Landrum, T. J., Lingo, A. S., \& Scott, T. M. (2011). Classroom misbehavior is predictable and preventable. Phi Delta Kappa, 93(2), 30-34. https://doi.org/10.1177/003172171109300207

LeBlanc, L., Swisher, R., Vitaro, F., \& Tremblay, R. E. (2007). School social climate and teachers' perceptions of classroom behavior problems: A 10 year longitudinal and multilevel study. Social Psychology of Education, 10(4), 429-442. https://doi.org/10.1007/s11218-007-9027-x

Lewis, R., Romi, S., Qui, X., \& Katz, Y. (2005). Teachers' classroom discipline in Australia, China and Israel. Teaching and Teacher Education, 21, 729-741. https://doi.org/10.1016/j.tate.2005.05.008

Lopes, J. \& Oliveira, C. (2016). Questionário - Indisciplina em Contexto de Sala de Aula. https://pt.surveymonkey.com/r/ZTW5DK6

Lopes, J., \& Santos, M. (2013). Teachers' beliefs, teachers' goals and teachers' classroom management: A study with primary teachers. Revista de Psicodidáctica, 18(1), 5-24. https://doi.org/10.1387/RevPsicodidact.4615

Lopes, E. M. (2006). Indisciplina em contexto educativo. Dissertação de Mestrado, Departamento de Educação e Psicologia, Universidade de Aveiro. RIA - Repositorio institucional: http://hdl.handle.net/10773/4988

Martin, N. K., \& Baldwin, B. (1994). Beliefs regarding classroom management style: Differences between novice and experienced teachers. Paper apresentado em Annual Conference of the Southwest Educational Research Association, San Antonio, TX, January, 1994. Recuperado de: https://files.eric.ed.gov/fulltext/ ED387471.pdf

Nash, P., Schlösser, A., \& Scarr, T. (2016). Teachers' perceptions of disruptive behaviour in schools: A psychological perspective. Emotional and Behavioural Difficulties, 21(2), 167-180. https://doi.org/10.1080/ 13632752.2015 .1054670

Özben, Ş. (2010). Teachers' strategies to cope with student misbehavior. Procedia-social and Behavioral Sciences, 2(2), 587-594. https://doi.org/10.1016/j.sbspro.2010.03.068

Piwowar, V., Thiel, F., \& Ophardt, D. (2013). Training in service teachers' competencies in classroom management. A quasi-experimental study with teachers of secondary schools. Teaching and Teacher Education, 30(1), 1-12. https://doi.org/10.1016/j.tate.2012.09.007 
Polat, S., Kaya, S., \& Akdağ, M. (2013). Investigating pre-service teachers' beliefs about classroom discipline. Kuramve Uygulamada Egitim Bilimleri, 13(2), 885-890. Recuperado de: https://files.eric.ed.gov/fulltext/ EJ1017368.pdf

Renca, A. A. (2008). A indisciplina na sala de aula: percepções de alunos e professores. Dissertação de Mestrado, Departamento de Educação e Psicologia, Universidade de Aveiro. RIA - Repositorio institucional: http://hdl.handle.net/10773/1045

Reynolds, K., Stephenson, J., \& Beaman, R. (2011). Teacher perceptions of non-compliance in rural primary schools in New South Wales. Education in Rural Australia, 21(2), 105-124. https://search.informit.com.au/ documentSummary; $\mathrm{dn}=775946750034706$; res=IELHSS

Robers, S., Zhang, J., \& Truman, J. (2012). Indicators of School Crime and Safety: 2011. (NCES 2012002/NCJ 236021). Washington, DC: National Center for Education Statistics, Bureau of Justice Statistics. Recuperado de: https://www.bjs.gov/content/pub/pdf/iscs11.pdf

Rothstein, R., Carnoy, M., \& Benveniste, L. (1999). Can Public Schools Learn from Private Schools? Case Studies in the Public \& Private Nonprofit Sectors. Washington, DC: Economic Policy Institute. Recuperado de: https://files.eric.ed.gov/fulltext/ED457293.pdf

Sandberg, J. F., \& Hoffert, S. L. (2001). Changes in children's time with parents: United States, 1981-1997. Demography, 38, 423-436. https://doi.org/10.1353/dem.2001.0031

Santos, J. S., \& Pascoinho, J. C. (2020). Prevenção da indisciplina num agrupamento de escolas de Portugal. Educação e Pesquisa, 46, e212779. https://doi.org/10.1590/S1678-4634202046212779

Simón, C., \& Alonso-Tapia, J. (2016). Positive classroom management: Effects of disruption management climate on behaviour and satisfaction with teacher. Revista de Psicodidactica, 21(1), 65-86. https://doi.org/10.1387/RevPsicodidact.13202

Singh, K., \& Billingsley, B. S. (1998). Professional support and its effects on teachers' commitment. The Journal of Educational Research, 91(4), 229-239. https://doi.org/10.1080/00220679809597548

Skaalvik, E. M., \& Skaalvik, S. (2008). Teacher self-efficacy: conceptual analysis and relations with teacher burnout and perceived school context. In R. Craven, H. W. Marsh, \& D. McInerney (Eds.), Self-processes, learning, and enabling human potential (pp. 223-247). Charlotte, NC: Information Age Publishing.

Skaalvik, E. M., \& Skaalvik, S. (2010). Teacher self-efficacy and teacher burnout: A study of relations. Teaching and teacher education, 26(4), 1059-1069. https://doi.org/10.1016/j.tate.2009.11.001

Skaalvik, E. M., \& Skaalvik, S. (2011). Teacher job satisfaction and motivation to leave the teaching profession: Relations with school context, feeling of belonging, and emotional exhaustion. Teaching and teacher education, 27(6), 1029-1038. https://doi.org/10.1016/j.tate.2011.04.001

Sun, R. C., \& Shek, D. T. (2012). Student classroom misbehavior: An exploratory study based on teachers' perceptions. The Scientific World Journal, 1-8. https://doi.org/10.1100/2012/208907

Tauber, R. (2007). Classroom management: Sound theory and effective practice. Westport, CT: Praeger.

Teixeira, C. M. (2007). Representações da indisciplina de professores do $3^{\circ}$ Ciclo do Ensino Básico (Estudo de caso). Dissertação de Mestrado, Universidade do Algarve. Sapientia - Repositorio de Universidade do Algarve: http://hdl.handle.net/10400.1/680

Tsouloupas, C., Carson, R. L., \& Matthews, R. (2014). Personal and school cultural factors associated with the perceptions of teachers' efficacy in handling student misbehavior. Psychology in the School, 51(2), 164180. https://doi.org/10.1002/pits

Tsouloupas, C. N., Carson, R. L., Matthews, R., Grawitch, M. J., \& Barber, L. K. (2010). Exploring the association between teachers' perceived student misbehaviour and emotional exhaustion: The importance of teacher efficacy beliefs and emotion regulation. Educational Psychology, 30(2), 173-189. https://doi.org/10.1080/01443410903494460 
Yilmaz, S., \& Sahinkaya, N. (2010). The relationship between the methods teachers use against the misbehaviour performed in the classroom and emphatic tendencies of teachers. Procedia Social and Behavioral Sciences, 2, 2932-2936. https://doi.org/10.1016/j.sbspro.2010.03.443

Fecha de recepción: 31 de julio de 2019. Fecha de revisión: 4 de mayo de 2020. Fecha de aceptación: 11 de mayo de 2020. Fecha de publicación: 1 de julio de 2020. 